NUP-A-96-12

\title{
Stationary Vortices of Elliptic Boundary in Drift and Geostrophic Shear Flows
}

\author{
Yoko KATO, Noriko KOIDE and Masatomo SATO* \\ Department of Physics, College of Science and Technology, \\ Nihon University,
}

\begin{abstract}
An analytic solution of the Charney-Hasegawa-Mima equaiton, a two-dimensional nonlinear equation describing geostrophic Rossby flow and plasma drift flow, is given which represents a stationary vortex of an elliptic boundary in a background flow having a linear shear. The solution is obtained through an appropriate ansatz for the arbitrary function of integration of the stationary equation. Three types of vortices emerge according to the chioce of parameters which characterize the distribution of vorticity: a generally asymmetric dipole inside the boudanry ellipse $E$, a monopole inside $E$, and a larger dipole lying across $E$.
\end{abstract}

KEYWORDS: elliptic-boundary vortex, drift-geostrophic shear flow, Charney-Hasegawa-Mima equation

\section{§1. Introduction}

This paper aims at giving a simple analytical solution for two-dimensional stationary vortices which are elongated in the direction of a background shear flow. Such vortices are often observed in natural atmospheric or oceanic flows on planets, as exemplified by the Great Red Spot of Jupiter and the Dark Spot of Neptune. Thus it is hoped that the solution will shed some light on understanding the dynamics of such elongated vortices.

The present analysis is based on the Charney-Hasegawa-Mima (CHM) equation. ${ }^{1,2)}$ It is a quasi-two-dimensional nonlinear equation for stream function commonly used to describe the geostrophic Rossby flow on a rotating planet as well as the electrostatic drift flow in a mangetized inhomogeneous plasma. Among many works devoted to the study of vortical motion described by the CHM and related equations, ${ }^{3)}$ the paper by Larichev and Reznik ${ }^{4}$

\footnotetext{
${ }^{*}$ E-mail: sato@gaia.phys.cst.nihon-u.ac.jp
} 
was the first that obtained an exact solution representing a steady dipolar vortex, usually called a modon, which moved with a constant velocity in a uniform flow. In their work, the entire plane was divided by a circular boundary into internal and external regions, and an appropriate linear equation for the stream function was solved in each region to obtain a vortical solution inside, and a rapidly decaying solution outside the circle; the two solutions were then connected on the boundary by requiring the continuty of the stream funciton and its first derivatives. This method of solution was applied by Horihata et al ${ }^{5)}$ to the case of background shear flows, and they obtained multipolar vortices inside the circular boundary for several kinds of shear flows. A more refined approach to the shear-flow case has been made by Moriguchi and Nozaki. ${ }^{6)}$ These and some other works ${ }^{3,7-11)}$ were motivated to give a monopolar vortex, with and without the background shear flow, to simulate the Jovian Red Spot.

On the other hand, there have been several works ${ }^{12-15)}$ which treated elliptic or elongated vortices in two-dimensional ordinary fluid described by the inviscid Navier-Stokes equation. In particular, in a study on trailing vortices behind a wing, Moore and Saffman ${ }^{12)}$ obtained steady exact solutions which consist of a monopolar core inside, and flows with uniform vorticity outside an ellipse. More recently, Boyd and $\mathrm{Ma}^{13)}$ studied numerically the elliptical modons in a uniform flow, while Nycander ${ }^{15)}$ considered the elongation of circular vortices in a background flow of uniform shear.

In the present paper, we deform the circular boundary used in previous works ${ }^{4-6,9,10)}$ into an ellipse, and consider a stationary vortex which moves with a constant velocity in the direction of the major axis. In order to facilitate the analysis in terms of elliptic coordinates, we take advantage of the arbitrariness of the funciton $F(\psi)$ of the stream funciton $\psi$ which appears in the integral of the stationary CHM equation. Thus by an appropriate ansatz for the function $F(\psi)$, we derive equations, one for each of the inner and outer regions, which are just the two-dimensional Poisson's equations with non-uniform vorticity and may be solved easily using the elliptic coordinates. ${ }^{16)}$ The solutions thus obtained represent monopolar as well as dipolar vortices in a background flow having an asymptotically linear shear, and may be considered as a generalization of the solutions by Moore and Saffman. ${ }^{12}$ )

The paper is organized as follows. In $\S 2$, the CHM equation is derived from the conservation of potential vorticity introducing the notations used, the boundary conditions on the ellipse $E$ are given, and the stationary CHM equations inside and outside $E$ are converted to the Poisson's equations. In $\S 3$, the solutions are obtained and the flow partterns are classified in three types according to the choice of parameters; brief accounts are also made for the case of uniform vorticity in each region and for the limiting case of a circular boundary. Finally, concluding remarks are given in $\S 4$. 


\section{§2. Basic Equations for Stationary Flow and Boundary Conditions}

The basic equations used in the present analysis are derived from the conservation of potential vorticity which holds for electrostatic drift flows as well as geostrophic Rossby flows provided that all the dissipative effects are ignored. The derivation ${ }^{3,11)}$ will be summarized below to explain relevant notations. For the drift flow, we assume a cold ion plasma in a uniform magnetic field along which electrons move freely to obey the Boltzmann distribution. As a result of charge neutrality, the ion density $n$ is then related to the electrostatic potential $\phi$ by $n=n_{0} \exp \left(e \phi / T_{e}\right)$, where $e$ is the elementary charge, $T_{e}$ the electron temperature and $n_{0}$ the ion density in the absence of $\phi$. Taking the $(x, y)$ coordinates in the plane perpendicular to magnetic field, we put $\ln n_{0}(y)=$ const. $+\beta y$, where $\beta$ is a constant. The potential vorticity is given by $q_{d}=\left(\Omega+\omega_{c i}\right) / n$, where $\Omega$ is the vorticity of the flow in the $(x, y)$ plane and $\omega_{c i}$ the cyclotron frequency of ions; we put $q=\ln q_{d}$ in the following. The varialbles are made dimensionless by the following substitutions:

$$
e \phi / T_{e} \rightarrow \phi, \quad \omega_{c i} t \rightarrow t, \quad(x, y) / \rho_{i} \rightarrow(x, y)
$$

where $\rho_{i}=\left(T_{e} / m_{i} \omega_{c i}^{2}\right)^{1 / 2}$, with $m_{i}$ the ion mass, is the ion Larmor radius at the electron temperature which we have assumed constant.

For the Rossby flow, we assume an incompressible shallow fluid in the geostrophic approximation. Taking the local Cartessian coordinates on the northern hemisphere of the planet with the $x$-axis toward east, the $y$-axis north and the $z$-axis local zenith, we express the Coriolis paramter $f=2 \omega \sin \alpha$, where $\omega$ is the spin angular velocity of the planet and $\alpha$ the latitude, as $f(y)=f_{0}+\beta y$ in the so-called $\beta$-plane approximation where $f_{0}$ and $\beta$ are constants. The depth of the atmosphere is expressed as $H=H_{0}+h$, where $h(x, y, t)$ is the displacement from the average depth $H_{0}$. The potential vorticity is $q_{R}=(\Omega+f) / H$, where $\Omega$ is the vorticity as above, and we put $q=q_{R}$ below. The dimensionless variables are defined by the substitutions

$$
h / H_{0} \rightarrow \phi, \quad f_{0} t \rightarrow t, \quad(x, y) / \rho_{R} \rightarrow(x, y)
$$

where $\rho_{R}=\sqrt{g H_{0}} / f_{0}$ is the Rossby radius with $g$ the gravitational acceleration.

Using the above definitions for the potential vorticity $q$ in each flow, the conservatin of $q$ is derived from the equations of continuty and ion motion for the drift flow, and from the conservation of mass in the depth $H$ and the geostrophic equations of motion for the Rossby flow, respectively. It is given by

$$
\left(\frac{\partial}{\partial t}+v \cdot \nabla\right) q=0
$$

where $\boldsymbol{v}$ is the flow velocity in the $(x, y)$ plane. 
In the following, we consider eq.(1) in the short-wavelength ordering which assumes $\nabla \sim$ $O(1)$ and $\partial / \partial t \sim \beta \sim O(\epsilon)$ in the dimensionless variables introduced above, where $\epsilon$ is a small paramter. Under this ordering, the function $\phi$ plays the role of a stream function, and the flow velocity $v$ is given by

$$
v_{x}=-\frac{\partial \phi}{\partial y}, \quad v_{y}=\frac{\partial \phi}{\partial x}
$$

and eq. (1) is expressed as ${ }^{5}$

$$
\frac{\partial}{\partial t} q+[\phi, q]=0
$$

where $[A, B]=\hat{z} \cdot(\nabla A \times \nabla B)$ is the Poisson bracket. The potential vorticity $q$ in eq. (3) is expressed as

$$
q=\nabla^{2} \phi-\phi+\beta y
$$

in the present short-wavelength ordering; eqs. (3) and (4) are the well-known CharneyHasegawa-Mima (CHM) equation. ${ }^{1,2)}$

As we consider a stationary vortex which propagates in the $x$ direction with a constant velocity $u$, we use a reference frame moving with the vortex. The space-time variables are transformed as $(x, y) \rightarrow(x-u t, y)$ and $t \rightarrow t$, but the variable $x$ will be used instead of $x-u t$ in the following for the sake of simplicity. The stream function in the moving frame is given by

$$
\psi(x, y)=\phi(x, y)+u y
$$

and the CHM equation (3) becomes

$$
[\phi+u y, q]=[\psi, q]=0
$$

for the stationary state. This equation is readily integrated to give

$$
q=F(\phi+u y)=F(\psi)
$$

where $F(\psi)$ is an arbitrary function of $\psi$.

Now we introduce an ellipse as the boundary curve which separates the entire $(x, y)$ plane into internal and external regions. ${ }^{12)}$ Let the equation of the ellipse $E$ be

$$
\frac{x^{2}}{a^{2}}+\frac{y^{2}}{b^{2}}=1
$$

where $a$ and $b$ are constants and $a>b$. In accordance with the elliptic boundary, we introduce the elliptic coordinates $(\xi, \eta)$ by

$$
x=c \cosh \xi \cos \eta, \quad y=c \sinh \xi \sin \eta,
$$


where $c=\left(a^{2}-b^{2}\right)^{1 / 2}$ is the distance from the origin to one of the foci of $E$. The curves $\xi=$ const. are a family of confocal ellipses, and one of them, $\xi=\xi_{0}$ say, coincides with $E$ provided that

$$
a=c \cosh \xi_{0}, \quad b=c \sinh \xi_{0},
$$

and the region $\xi<\xi_{0}\left(\xi>\xi_{0}\right)$ corresponds to the inside (outside) of $E$. Then the boundary conditions on $\psi$ at the boundary $E$ are given by

$$
\psi=0 \quad \text { at } \xi=\xi_{0}
$$

and

$$
\frac{\partial \psi_{i n}}{\partial \xi}=\frac{\partial \psi_{e x}}{\partial \xi} \quad \text { at } \quad \xi=\xi_{0}
$$

where $\psi_{\text {in }}$ and $\psi_{e x}$ signify, respectively, the solution inside and outside $E$. Condition (11) follows from the requirement that the boundary curve itself must be one of the stream lines, $\psi=$ const, and the arbitrary constant of $\psi$ has been chosen to be null on $E$. Condition (12), on the other hand, comes from the continuity of tangential velocity across $E$; another condition $\partial \psi / \partial \eta=0$ at $\xi=\xi_{0}$, expressing the vanishing of the velocity normal to $E$, is automatically satisfied by virtue of condition (11).

We proceed to specify the arbitrary function $F(\psi)$ in eq. (7). In the case of the modon with a circular boundary first derived by Larichev and Reznik, ${ }^{4}$ ) the function $F$ in the external region was chosen to yield a highly localized solution $\phi$, decaying nearly exponentially at infinity, and the resulting equation for $\phi$ was simple enough to allow an analytic solution. In the present case of elliptic boundary, the use of elliptic coordinates makes difficult to obtain highly localized solutions in a simple way. We therefore choose the function $F(\psi)$ to yield equations which allow simple analytic solutions. Specifically, we assume ${ }^{16)}$

$$
F(\psi)= \begin{cases}-\psi+\omega_{1}: & \text { inside } E, \\ -\psi+\omega_{0}: & \text { outside } E,\end{cases}
$$

where $\omega_{1}$ and $\omega_{0}$ are constants and $\omega_{1} \neq \omega_{0}$ in general. Then eq. (7) becomes

$$
\begin{aligned}
& \nabla^{2} \psi=-(u+\beta) y+\omega_{1}: \text { inside } E \\
& \nabla^{2} \psi=-(u+\beta) y+\omega_{0}: \text { outside } E
\end{aligned}
$$

The solutions derived from eqs. (14) will be given and discussed in the next section.

\section{§3. Stationary Solutions}

For the sake of convenience, we distinguish the cases $u+\beta \neq 0$ and $u+\beta=0$. The solution in the limiting case of a circular boundary is also presented for comparison. 


\subsection{The case of $u \neq-\beta$.}

From (14-1) and the boundary condition (11), the internal solution is determined to be

$$
\psi_{i n}=A\left(\frac{x^{2}}{a^{2}}+\frac{y^{2}}{b^{2}}-1\right)(y+B)
$$

with

and

$$
A=-\frac{1}{2} \frac{a^{2} b^{2}}{3 a^{2}+b^{2}}(u+\beta)
$$

$$
B=-\frac{3 a^{2}+b^{2}}{a^{2}+b^{2}} \frac{\omega_{1}}{u+\beta}
$$

In terms of the elliptic coordinates (9), the internal solution may be expressed also as follows,

$$
\psi_{\text {in }}=F_{0}(\xi)+F_{1}(\xi) \sin \eta+F_{2}(\xi) \cos 2 \eta+F_{3}(\xi) \sin 3 \eta
$$

where $F_{n}(\xi), n=0,1,2,3$, are given by

$$
\begin{aligned}
& F_{0}(\xi)=-\frac{1}{8} \omega_{1}\left\{\left(a^{2}+b^{2}\right)-c^{2} \cosh 2 \xi\right\} \\
& F_{1}(\xi)=\frac{1}{32}(u+\beta) c\left\{\left(3 a^{2}+b^{2}\right) \sinh \xi-c^{2} \sinh 3 \xi\right\} \\
& F_{2}(\xi)=-\frac{c^{2}}{a^{2}+b^{2}} F_{0}(\xi) \\
& F_{3}(\xi)=-\frac{c^{2}}{3 a^{2}+b^{2}} F_{1}(\xi),
\end{aligned}
$$

and $F_{n}\left(\xi_{0}\right)=0$ in accordance with the boundary condition (11). In case of $|B|<b$, this solution vanishes on the line $y=-B$ in addition to the boundary $E$.

We next treat the solution in the external region using the elliptic coordinates. Since the two-dimensional Laplacian is transformed to

$$
\nabla^{2}=\frac{2}{c^{2}(\cosh 2 \xi-\cos 2 \eta)}\left(\frac{\partial^{2}}{\partial \xi^{2}}+\frac{\partial^{2}}{\partial \eta^{2}}\right)
$$

in the elliptic coordinates, eq. (14-2) becomes

$$
\begin{aligned}
\left(\frac{\partial^{2}}{\partial \xi^{2}}+\frac{\partial^{2}}{\partial \eta^{2}}\right) \psi= & \frac{1}{2} \omega_{0} c^{2}(\cosh 2 \xi-\cos 2 \eta) \\
& -\frac{1}{4}(u+\beta) c^{3}(\sinh 3 \xi \sin \eta-\sinh \xi \sin 3 \eta)
\end{aligned}
$$

We then seek the solution of eq. (21) in the form

$$
\psi_{e x}=G_{0}(\xi)+G_{1}(\xi) \sin \eta+G_{2}(\xi) \cos 2 \eta+G_{3}(\xi) \sin 3 \eta .
$$

The equations for $G_{n}(\xi), n=0,1,2,3$, are derived by inserting this expression for $\psi_{e x}$ in the left-hand side of eq. (21) and comparing the coefficients of $1, \sin \eta, \cos 2 \eta$ and $\sin 3 \eta$ on both sides. According to conditions (11) and (12), the functions $G_{n}(\xi)$ must satisfy the conditions

$$
G_{n}\left(\xi_{0}\right)=0 \quad \text { and } \quad G_{n}^{\prime}\left(\xi_{0}\right)=F_{n}^{\prime}\left(\xi_{0}\right), \quad(n=0,1,2,3)
$$


and the results are given as follows,

$$
\begin{aligned}
G_{0}(\xi)= & \frac{1}{2}\left(\omega_{1}-\omega_{0}\right) a b\left(\xi-\xi_{0}\right)-\frac{1}{8} \omega_{0}\left\{\left(a^{2}+b^{2}\right)-c^{2} \cosh 2 \xi\right\} \\
G_{1}(\xi)= & F_{1}(\xi) \\
G_{2}(\xi)= & \frac{1}{8} \omega_{0} c^{2}-\frac{1}{4}\left(\omega_{1}-\omega_{0}\right) a b \sinh 2 \xi \\
& +\frac{1}{2}\left\{\frac{a^{2} b^{2}}{a^{2}+b^{2}} \omega_{1}-\frac{1}{4}\left(a^{2}+b^{2}\right) \omega_{0}\right\} \cosh 2 \xi \\
G_{3}(\xi)= & F_{3}(\xi) .
\end{aligned}
$$

Now we examine the obtained results in some detail. In general, we may call the flow region with closed stream lines the vortex core, or simply the vortex, and the one with open stream lines extending to infinity the background flow. Then it is apparent that the internal solution (15) always represents a vortex, while the external solution a background linear shear flow, $\psi \propto y^{3}$, at large distances from the $x$-axis. It is also possible, however, that closed stream lines do appear in the external region adjacent to $E$ for some choice of the parameters involved. It turns out that the stationary flow patterns obtained here may be classified to three types, I, IIa and IIb, as follows:

(I) The case of $|B|<b: A$ dipolar vortex inside $E$.

The line $y=-B$ lies inside the ellipse $E$ for $|B|<b$, and $\psi_{\text {in }}=$ const. represents a dipolar vortex consisting of two vortices of opposite polarities; see Figs. 1 . Since $B$ (that is, $\omega_{1}$ ) is small, the vorticity inside depends rather strongly on $y$. In the special case of $\omega_{1}=0$ $(B=0)$, the two vortices have the some size and form a modon compressed into an ellipse (Fig. 1(ii)). The stream lines outside $E$ are all open, and not symmetiric with respect to the $x$-axis for $u+\beta \neq 0$ under consideration.

(II) The case of $|B| \geq b$.

The line $y=-B$ is outside the ellipse $E$ for $|B|>b$, or tangent to $E$ for $|B|=b$, and the internal region is occupied by a monopolar vortex. Then the background flow and the vortical flow meet with opposite directions each other in a outer region near $E$, and the external stream lines are arranged to avoid the resulting singularity. There are two possible types:

(IIa) A monopolar vortex inside $E$ (Figs. 2).

The singularity mentioned above is avoided by forming a stagnation point on the $y$-axis. Thus almost all the external stream lines, except those few very close to $E$, are all open, and the only vortex is the monopolar one mainly consisting of the internal stream lines. We note is passing that Fig. 2(i) is drawn for $\omega_{0}=\omega_{1}=-0.5$, that is, for a special case where the external solution coinsides with the internal one.

(IIb) A "big" dipolar vortex across $E$ (Figs. 3). 
A monopolar vortex of polarity opposite to the internal one is formed in the outer region adjacent to $E$, thus forming a "big" dipolar vortex together with the internal one. In this case, two stagnation points are formed on either end of the external vortex. If the outer vortex is small and weak, the pattern would look like the monopolar vortex of case IIa.

Next, we consider the parameter range for the three flow patterns described above. In what follows, we put

$$
\lambda=u+\beta
$$

for the coefficient of linear vorticity. Then the sets of parameters involved in the present stationary solution are $(a, b)$ and $\left(\lambda, \omega_{1}, \omega_{0}\right)$ determining, respectively, the ellipse $E$ and the distribution of vorticity. We shall fix the boundary $E$ by setting $a=2$ and $b=1$ (or $c=\sqrt{3}$ and $\xi_{0}=0.5493$ ), which have been used throughout in Figs. 1 to 3 .

An example of the flow pattern diagram on the $\left(\omega_{0}, \omega_{1}\right)$ plane is shown in Fig. 4 for the particular case of $\lambda=-0.5$. (As a result of the symmetry property of eqs. (14) to be mentioned below, we need consider only the negative values of $\lambda$.) A dipolar vortex of type I belongs to the domain between the two straight lines running parallel to the abscissa; these lines correspond to $|B|=b$, that is, to $\omega_{1}= \pm|\lambda|\left(a^{2}+b^{2}\right) b /\left(3 a^{2}+b^{2}\right)= \pm \frac{5}{14}|\lambda|$. A monopolar vortex of type Ila emerges in the domain, for either sign of $\omega_{1}$, between the straight line just mentioned and a curve above (or, below) it. Finally, a dipolar vortex of case IIb belongs to the domains outside the curve.

When the parameter $\lambda$ is changed, the curve dividing the two types IIa and IIb moves as depicted in Fig. 5 where only the lower half plane $\left(\omega_{1} \leq 0\right)$ is shown and the straight line, $\left|\omega_{1}\right|=(5 / 14)|\lambda|$, is omitted. As can be seen from Fig. 4, the flow pattern is symmetric with respect to the origin of the $\left(\omega_{0}, \omega_{1}\right)$ plane for a fixed value of $\lambda$.

In the present solution, similar distributions of stream lines may be obtained by changing the $\operatorname{sign}(\mathrm{s})$ of some of the parameters $\left(\lambda, \omega_{1}, \omega_{0}\right)$, keeping their magnitude unchanged. From eqs. (14-1) and (14-2), it can be shown that given a flow pattern with a particular set of paramters $\left(\lambda, \omega_{1}, \omega_{0}\right)$, the patterns with $\left(-\lambda,-\omega_{1}, \omega_{0}\right),\left(\lambda,-\omega_{1},-\omega_{0}\right)$ and $\left(-\lambda,-\omega_{1},-\omega_{0}\right)$ are similar to it as follows:

- $\left(-\lambda, \omega_{1}, \omega_{0}\right)$ : The distribution of stream lines is obtained by replacing $y$ by $-y$. The sign of $\psi$ is unchanged, and the direction of the corresponding vortex and background flow is the same as the original one.

- $\left(\lambda,-\omega_{1},-\omega_{0}\right)$ : The distribution of stream lines is obtained by replacing $y$ by $-y$ and by changing the sign of $\psi$. The direction of the corresponding vortex and background flow is reversed.

- $\left(-\lambda,-\omega_{1},-\omega_{0}\right)$ : The disctibution of stream lines is unchanged but the direction of flow is reversed. 
Thus it is sufficient ot consider only the case of negative $\lambda$ as shown in Figs. 4 and 5 .

We remark finally that the velocity $u$ of the moving frame and the parameter $\beta$ do not appear separately in the solution, but always as the combination $\lambda=u+\beta$. On the other hand, it is well known ${ }^{3)}$ that if $u$ becomes equal to the phase velocity of linear drift-Rossby waves given by $V_{p h}=-\beta /\left(1+k^{2}\right)$, where $k$ is the non-dimensional wave number, then the vortex may lose energy by radiating the waves. This argument will limit the possible range of $\lambda$ to $\lambda<0$ or $\lambda>\beta$.

\subsection{The case of $u=-\beta$}

As mentioned just above, this case had been better excluded since it would correspond to a vortex moving with the long wavelength limit $(k \rightarrow 0)$ of the linear phase velocity. For the sake of completeness, however, a brief account of the result is given below.

Putting $u+\beta=0$ in eqs. (14), we have

$$
\begin{aligned}
& \nabla^{2} \psi=\omega_{1}: \text { inside } E \\
& \nabla^{2} \psi=\omega_{0}: \text { outside } E
\end{aligned}
$$

which are the same as the constant vorticity equations in the two-dimensional Navier-Stokes flow. The boundary conditions are given by eqs. (11) and (12) as before.

The internal solution is given by

$$
\psi_{\text {in }}=A_{1}\left(\frac{x^{2}}{a^{2}}+\frac{y^{2}}{b^{2}}-1\right), \quad A_{1}=\frac{a^{2} b^{2}}{2\left(a^{2}+b^{2}\right)} \omega_{1}
$$

corresponding to con-centric ellipses, and the external solution by

$$
\psi_{e x}=G_{0}(\xi)+G_{2}(\xi) \cos 2 \eta
$$

where the functions $G_{0}(\xi)$ and $G_{2}(\xi)$ are the same as given by eqs. (24). Apart from notations, these solutions have been obtained by Moore and Saffman ${ }^{12)}$ for a line vortex in an imposed strain.

While the internal solution (27) represents always a monopolar vortex, the external flow may have different structures according to the choice of parameters $(a, b)$ and $\left(\omega_{1}, \omega_{0}\right)$. This can be seen by the asymptotic form of $\psi_{e x}$ for $\xi \gg 1$. It is given by

$$
\psi_{e x} \simeq \frac{1}{2(a+b)}\left\{\left[b \omega_{0}-\frac{a b(a-b)}{a^{2}+b^{2}} \omega_{1}\right] x^{2}+\left[a \omega_{0}+\frac{a b(a-b)}{a^{2}+b^{2}} \omega_{1}\right] y^{2}\right\} .
$$

The case of practical interest will be the one with the same sign of $\omega_{1}$ and $\omega_{0}$. Then the external stream lines are either closed forming a large elliptical vortex together with the internal ones for $\omega_{1} / \omega_{0}<\left(a^{2}+b^{2}\right) / a(a-b)$, or open forming hyperbolae at far distance for $\omega_{1} / \omega_{0}>\left(a^{2}+b^{2}\right) / a(a-b)$. In the marginal case $\omega_{1} / \omega_{0}=\left(a^{2}+b^{2}\right) / a(a-b)$, the stream lines are parallel to the $x$-axis at infinity where $\psi_{\text {ex }}=\frac{1}{2} \omega_{0} y^{2}$. 


\subsection{The case of circular boundary}

When the boundary ellipse $E$ degenerates to a circle with the radius $r_{0}$, eqs. (14-1) and (14-2) may be solved easily in terms of the polar coordinates $(r, \theta)$ defined by $x=r \cos \theta$ and $y=r \sin \theta$. The boundary coditions (11) and (12) are simply replaced by $\psi=0$ and the continuty of $\partial \psi / \partial r$ at $r=r_{0}$. The resulting solution is given as follows:

$$
\psi_{\text {in }}=-\frac{1}{8}(u+\beta)\left(r^{2}-r_{0}^{2}\right) r \sin \theta+\frac{1}{4} \omega_{1}\left(r^{2}-r_{0}^{2}\right), \quad r<r_{0}
$$

and

$$
\begin{aligned}
\psi_{e x}= & -\frac{1}{8}(u+\beta)\left(r^{2}-r_{0}^{2}\right) r \sin \theta+\frac{1}{4} \omega_{0}\left(r^{2}-r_{0}^{2}\right) \\
& +\frac{1}{2}\left(\omega_{1}-\omega_{0}\right) r_{0}^{2} \ln \left(r / r_{0}\right) . \quad r>r_{0}
\end{aligned}
$$

These expressions are to be compared with eqs. (15)-(19) and eqs. (22) and (24), respectively. Since the cubic terms are contained in the form $r^{2} y$ in $\psi_{i n}$, the solution depends on $\theta$ only through $\sin \theta$.

\section{§4. Concluding Remarks}

In this paper, we have shown that analytical solutions for stationary vortices with elliptic boundary, decsribed by the Charney-Hasegawa-Mima equation, may be obtained by suitably choosing the arbitrary function of integration for the stationary equation. The vortices are present in a background shear flow in agreement with the physical understanding that the vortices are fed energy and elongated by the background flow.

When the propagation velocity of vortices $(u)$ does not coincide with the long-wavelength limit of the phase velocity of linear waves $(-\beta)$, the background flow has a linear shear. The vortex core obtained in this case consists of a dipole or monopole inside the boundary ellipse, or a larger dipole made up of one monopole inside and another outside the ellipse; which is the case depends on the choice of parameters characterizing the distribution of vorticity. Although it turns out that a genuine monopolar vortex is obtained only for a limited range of the parameters, it will be also possible that an asymmetric dipole having one of the eddies much greater than the other would look like a monopole, thus extending the parameter range for an apparent monopolar vortex.

The realization of a stationary solution will be determined by its stability against perturbations which is not considerd in the present paper. It is physically plausible that a vortex in an external shear flow will be more stable when the shear and the vorticity anormaly have the same sign than the opposite case, as investigated partly by Moore and Saffman ${ }^{12)}$ for the two-dimensional Navier-Stokes flow of uniform external shear. This suggests that some or many of the solutions (with $u \neq-\beta$ ) obtained above would be vulnerable to perturbations 
since they have neighbouring stream lines running in opposite directions near the boundary ellipse. On the other hand, the stationary equations for the case $u=-\beta$ reduce formally to the uniform shear case of Moore and Saffman and the resulting elliptic vortices could be stable if the uniform vorticities inside and outside have the same sign. However, the solutions in this case have another difficulty of radiating linear waves as mentioned earlier.

Then, the tasks in the future will be first to examine the stability of the solutions obtained above, and to seek the possibility of finding out elliptic or elongated monopolar vortices of the stationary Charney-Hasegawa-Mima equation in an external flow of uniform shear.

\section{Acknowledgement}

The authors are deeply indebted to Professor Yoshio Ishikawa, Department of Aerospace Engineering, College of Science and Technology, Nihon University, for getting the paper by Moore and Saffman.

1) J. G. Charney: Geophys. Public. Kosjones Nors. Videnshap. Akad. Oslo 17(1948)3.

2) A. Hasegawa and K. Mima: Phys. Fluids 21(1978)87.

3) For a recent review article, see J. Nycander: Chaos 4(1994)253.

4) V. D. Larichev and G. M. Reznik: Dokl. Akad. Nauk SSSR 231(1976)1077.

5) S. Horihata, H. Irie and M. Sato: J. Phys. Soc. Jpn. 59(1990) 1242.

6) H. Moriguchi and K. Nozaki: J. Phys. Soc. Jpn. 61(1992) 117.

7) T. Maxworthy and L. G. Redekopp: ICARUS 29(1976)261.

8) L. G. Redekopp: J. Fluid Mech. 82(1977)725.

9) J. Nycander: J. Plasma Phys. 39(1988)413.

10) J. Nycander: Nonlinear World, ed. V. G. Bar'yakhtar (World Scientific, Singapore, 1989) Vol 2, p.933.

11) K. Orito, M. Sato and H. Irie: J. Phys. Soc. Jpn. 64(1995)1874.

12) D. W. Moore and P. G. Saffman: Aircraft Wake Turbulence and Detection, ed. J. H. Olsen, A. Goldberg and M. Rogers (Plenum Press, New York, 1971) p.339.

13) S. Kida: J. Phys. Soc. Jpn. 50(1981)3517.

14) J. P. Boyd and H. Ma: J. Fluid Mech. 221(1990)597.

15) J. Nycander: J. Fluid Mech. 287(1995) 119.

16) Y. Kato and M. Sato: J. Phys. Soc. Jpn. 65(1996)899. 

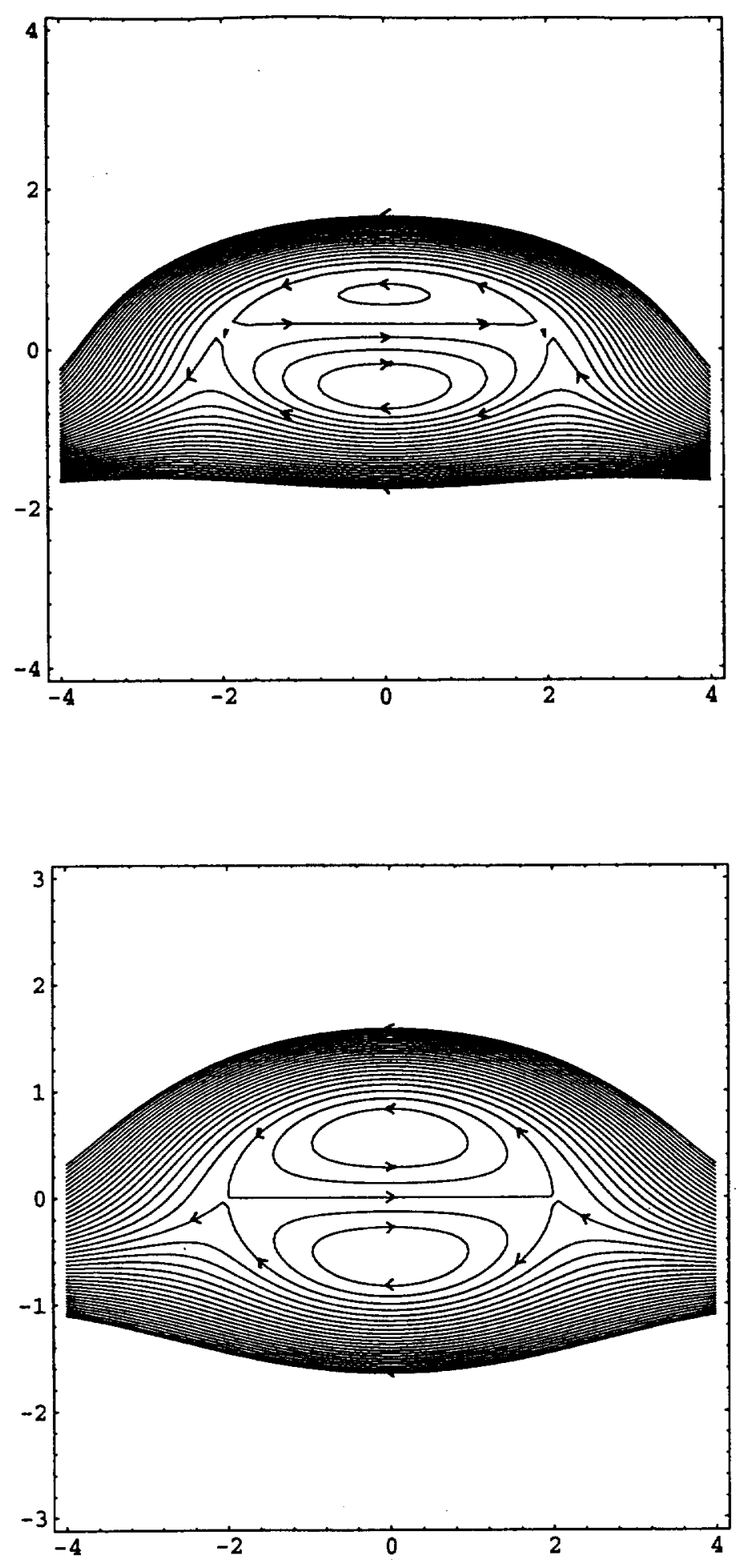

Fig. 1 Stream lines, $\psi=$ const., in the moving frame for type I: a dipolar vortex inside the boundary ellipse $E$. In all the figures below, the major and minor radii of $E$ are $a=2$ and $b=1$, respectively, corresponding to $c=\sqrt{3}$ and $\xi_{0}=0.5493$ (cf. eq. (10)).

(i) An asymmetric dipolar vortex: $u+\beta=-0.4, \omega_{0}=0.2$ and $\omega_{1}=-0.05$. The interval of stream lines is 0.01 .

(ii) An elongated modon: $u+\beta=-0.5, \omega_{0}=0.1$ and $\omega_{1}=0$. The interval of stream lines is 0.01 . The scale is taken different for $x$ and $y$ axes in this particular figure. 

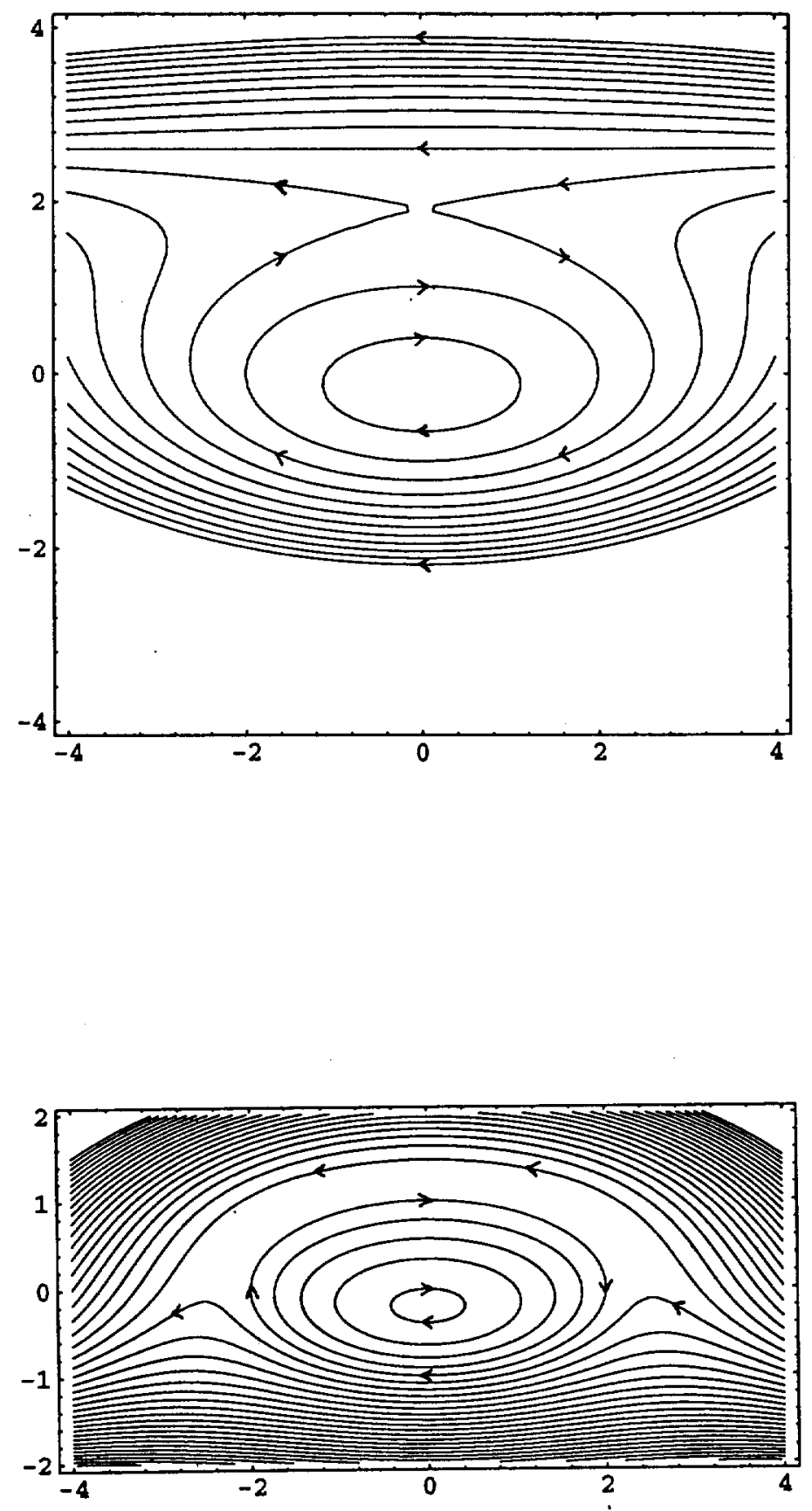

Fig.2 Stream lines, $\psi=$ const., in the moving frame for type Ila: a monopolar vortex inside the boundary ellipse $(a=2, b=1)$.

(i) $u+\beta=-0.5$ and $\omega_{0}=\omega_{1}=-0.5$. The interval of stream lines is 0.1406 and $\psi=-0.1406$ at the stagnation point. In this case, the inner and outer solutions are the same.

(ii) $u+\beta=-0.6, \omega_{0}=0.2$ and $\omega_{1}=-0.5$. The interval of stream lines is 0.04 . The stagnation point on the $y$-axis is not shown. 

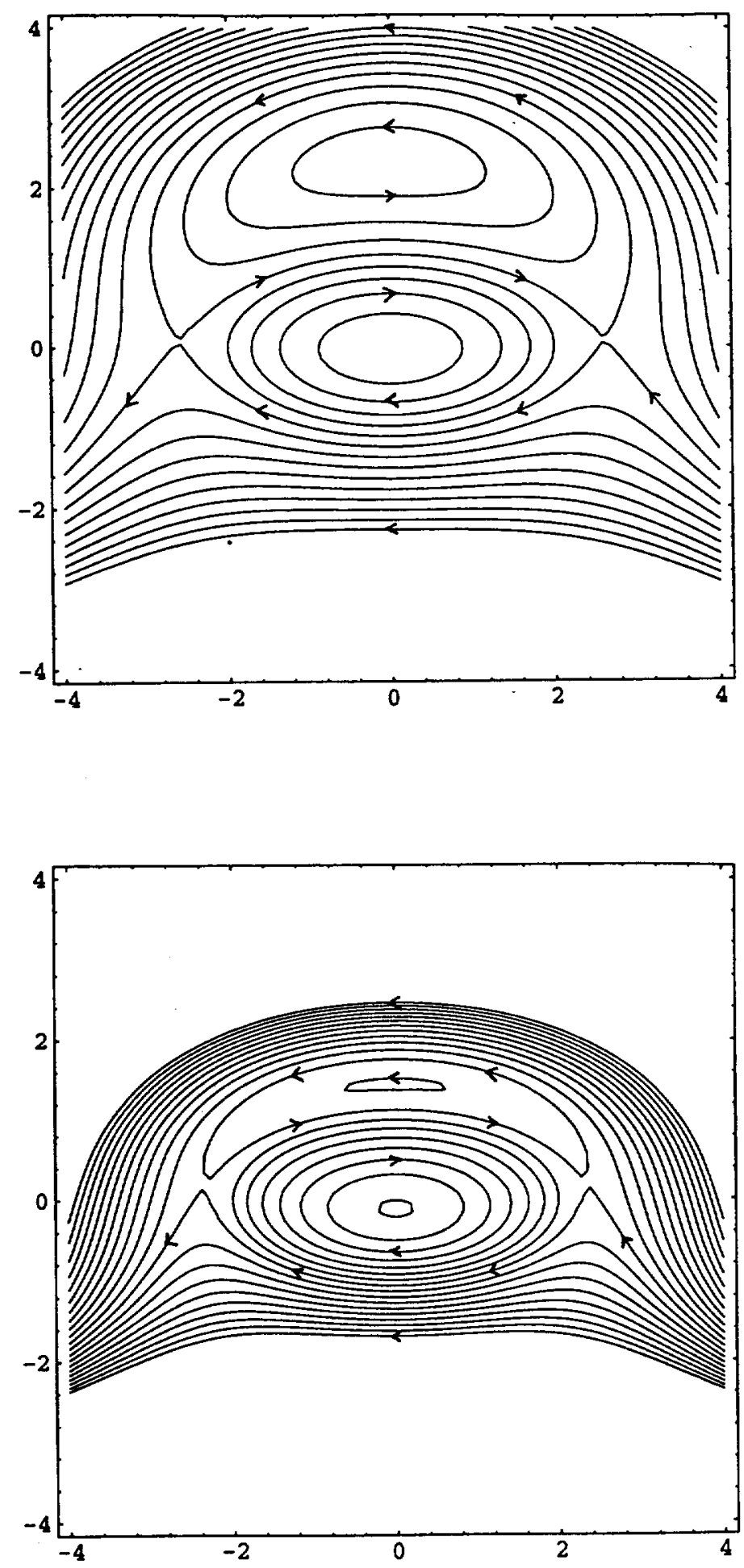

Fig.3 Stream lines, $\psi=$ const., in the moving frame for type IIb: a "big" dipolar vortex across the boundary ellipse $(a=2, b=1)$.

(i) $u+\beta=-0.1, \omega=0.1$ and $\omega_{1}=-0.5$. The interval of stream lines is 0.05374 and $\psi=-0.05374$ at the stagnation points.

(ii) $u+\beta=-0.3, \omega_{0}=0.3$ and $\omega_{1}=-0.5$. The interval of stream lines is 0.03336 and $\psi=-0.03336$ at the stagnation points. 


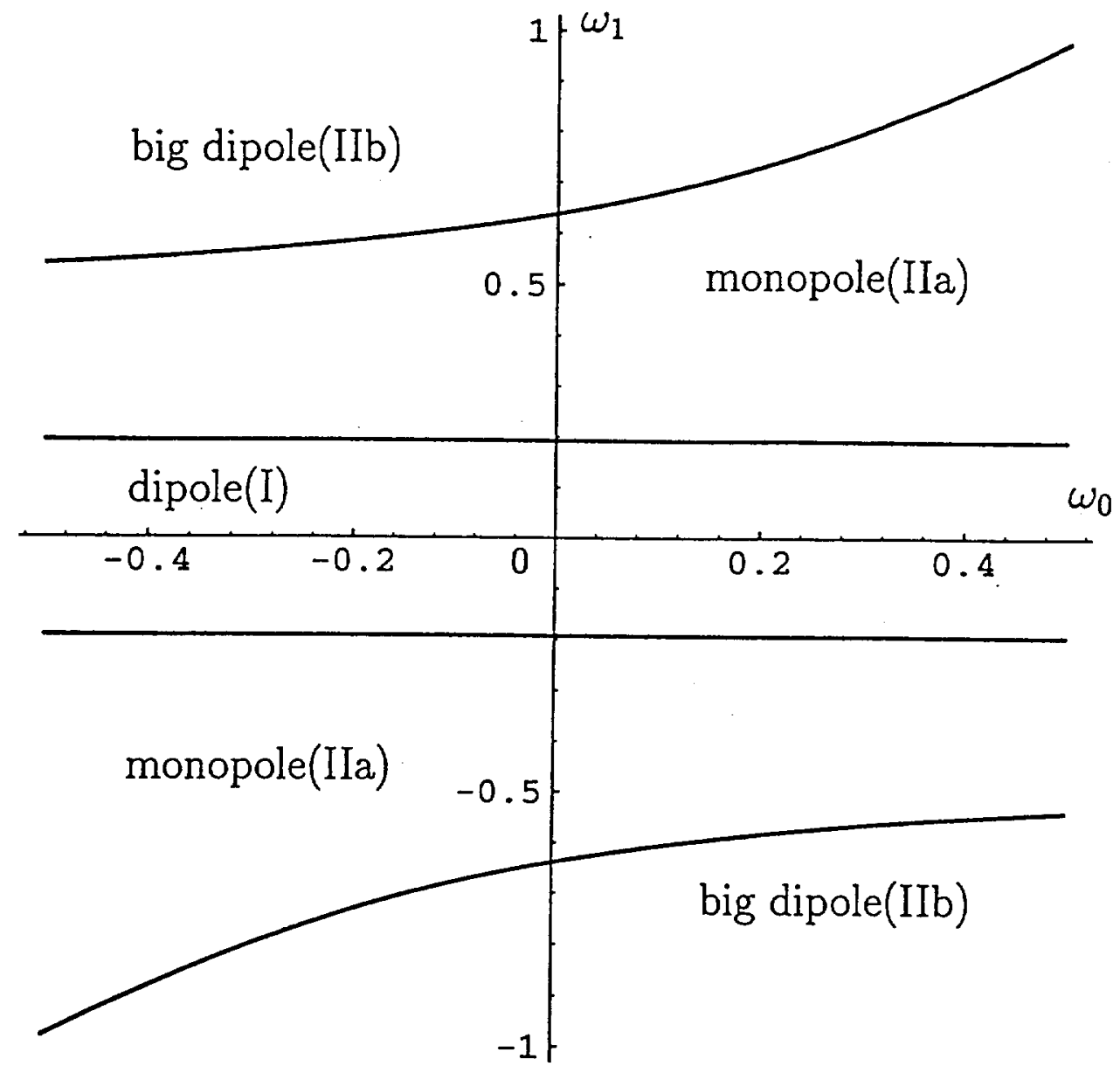

Fig.4 Vortex patterns on the $\left(\omega_{0}, \omega_{1}\right)$ plane in the case of $a=2, b=1$ and $\lambda \equiv u+\beta=-0.5$. The two straight lines parallel to the abscissa are given by $\omega_{1}= \pm|\lambda|\left(a^{2}+b^{2}\right) b /\left(3 a^{2}+b^{2}\right)$, corresponding to $|B|=b$ (cf. eq. (17)). 


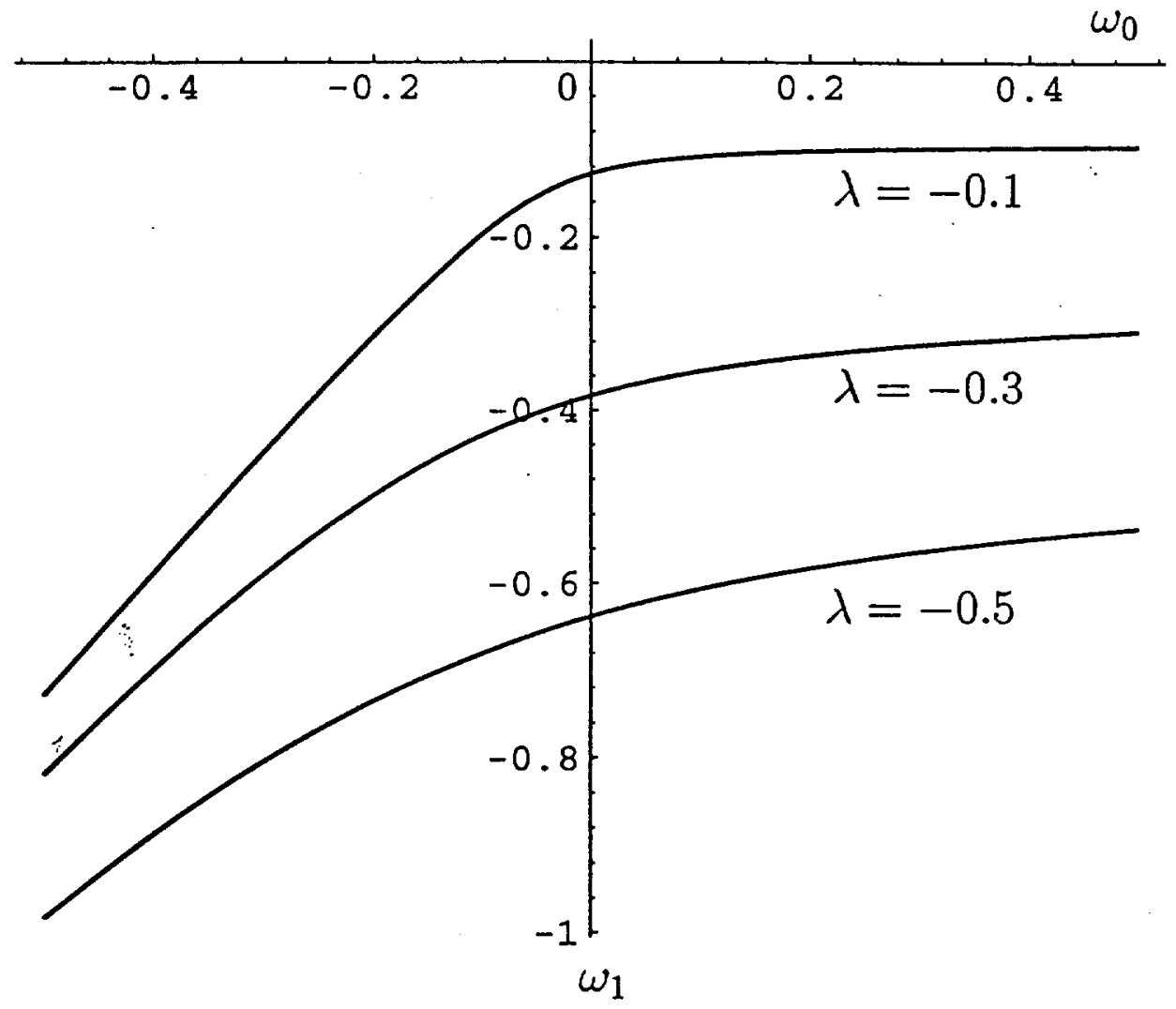

Fig.5 The movement of the border between the monopolar pattern IIa and the "big" dipolar pattern IIb as $\lambda$ is changed ( $a=2, b=1)$. Only the case of negative $\lambda$ is shown, the figure being symmetric with respect to the origin for positive $\lambda$. 